\title{
Verhaltenstherapeutische Behandlung eines Patienten mit beginnender Alzheimer-Demenz
}

\author{
Behavioral Treatment of a Patient with Early-Stage Alzheimer's Disease
}

\author{
T. Ehrhardt A. Plattner F. Padberg H.-J . Möller H. Hampel
}

Station D2, Forschungsgruppe dementielle Erkrankungen, Gedächtnissprechstunde, Psychiatrische Klinik und Poliklinik der LudwigMaximilians-Universität München

\section{Schlüsselwörter}

Alzheimer Demenz · Verhaltenstherapeutisches Kompetenztraining (VKT)

\section{Zusammenfassung}

Der folgende Fall berichtet von einer verhaltenstherapeutischen Behandlung eines 53jährigen Patienten mit einer beginnenden Alzheimer Demenz (AD). Die angewandte Methode basiert auf dem von Ehrhardt und Mitarbeitern entwickelten Verhaltenstherapeutischen Kompetenztraining (VKT) für Patienten mit einer AD im Anfangsstadium. Dabei finden in etwa 20 Sitzungen eine Aktivierung der vorhandenen Ressourcen des Patienten und eine Unterstützung bei der Bewältigung der Erkrankung statt. Der günstige Therapieverlauf wird zum einen deskriptiv und zum anderen mit Hilfe kognitiver Leistungstests veranschaulicht. In der Diskussion werden neben einer kritischen Reflexion der Therapie und der Methode auch Hinweise über den eigentlichen Fall hinaus auf den aktuellen Stand der Diskussion zur psychologischen Behandlung der AD gegeben.

\section{Einleitung}

Wegen der erwarteten Zunahme an Patienten mit A Izheimer Demenz (AD) in den nächsten Jahrzehnten droht eine Kostenexplosion in der $\mathrm{G}$ esundheitsversorgung. $\mathrm{E}$ in dringendes gesundheitspolitisches A nliegen ist daher die Entwicklung und $E$ valuation rehabilitativer $M$ aßnahmen, die in der Lage sind, die Pflegebedürftigkeit der Betroffenen zu vermeiden oder ihr E intreten zumindest aufzuschieben. G eeignete $M$ aßnahmen bestehen in einer Verlangsamung des fortschreiten-

\section{Key Words \\ Alzheimer's disease · Behavioral therapy · Behavioral Competency Training (VKT)}

\section{Summary}

The following case report shows a behavioral treatment of a 53-year-old Patient with Alzheimer's Disease (AD). The treatment includes procedures described in the Behavioral Competency Training (VKT) for patients with early-stage AD developed by Ehrhardt and associates. The program consists of about 20 weekly treatment sessions in which the patient's resources are activated and patient's coping is supported. The therapy and the intervention are debated in the light of the ongoing discussion about a state-of-the-art therapy for AD.

\begin{tabular}{ll}
\hline KARGER & ○ 1999 S. K arger G mbH , F reiburg \\
Fax +49 761 45207 14 & A ccessible online at: \\
$\begin{array}{l}\text { E-mail kargergmbh@aol.com } \\
\text { www.karger.com }\end{array}$ & http://B ioM edN et.com/karger
\end{tabular}

den kognitiven A bbaus, aber auch in einer günstigen B eeinflussung von Stimmungsveränderungen und Verhaltensproblemen.

E s gibt B elege, daß ein Nootropikum in Kombination mit einem neuropsychologischen Training bedeutsam größere E ffekte auf kognitive Parameter hatte als das Nootropikum alleine [H eiss et al., 1994; Yesavage et al., 1981]. A m Training isolierter kognitiver F unktionen wird allerdings immer wieder kritisiert, daß eine $\mathrm{G}$ eneralisierung der trainierten Techniken und Ü bungen auf nichttrainierte Bereiche und somit die A II- 
tagsrelevanz der Trainings nicht nachgewiesen werden kann [Q uayhagen und Q uayhagen, 1989; Y esavage et al., 1981].

$\mathrm{E}$ in weiterer schwerwiegender K ritikpunkt an Gedächtnistrainingsprogrammen ist die zu deutliche Konfrontation mit den wachsenden kognitiven D efiziten und den Depressionen und Verhaltensproblemen, die daraus resultieren können [B aines et al., 1987; Scanland und E mershaw, 1993]. D epressive Verstimmungen wirken sich zusätzlich negativ auf die generelle kognitive L eistungsfähigkeit der Patienten aus. Vor allem bei der A ufnahme von Information spielen psychologische Parameter, wie E instellung, Interesse und A ufmerksamkeit eine große R olle [M arkowitsch, 1992]. D epressionen und vor allem Verhaltensprobleme von A D-Patienten erhöhen das Risiko, daß auch die pflegenden A ngehörigen eine behandlungsbedürftige depressive $E$ rkrankung entwickeln, und führen dazu, daß die Patienten frühzeitig institutionalisiert werden [R ubin und K inscherf, 1989].

Vor allem immanente M erkmale der E rkrankung, wie ein bereits prämorbid niedriges A ktivitätsniveau [B roe et al., 1990; Friedland et al, 1996], dysfunktionales Coping [M otomura et al., 1996], Partnerschaftskonflikte [B auer et al., 1994] und die hohe Prävalenz depressiver Symptome zu B eginn der E rkrankung [Patterson und B olger, 1994], weisen den Weg zu einem psychotherapeutischen A nsatz. D ie Pathogenese der dementiellen Symptomatik ist zwar auf die degenerativen Veränderungen in der $\mathrm{H}$ irnrinde zurückzuführen, Auftreten und Schwere der kognitiven Symptome können aber von Faktoren wie emotionaler Zustand und allgemeiner A ktivierung moduliert werden. Das emotionale Wohlbefinden von ADPatienten kann durch eine $V$ ielzahl von verhaltenstherapeutischen Techniken wie A ktivitätenaufbau, Kognitive Techniken und die U nterstützung einer emotionalen Bewältigung verbessert werden.

Für Patienten mit einer A D im Frühstadium liegen nur vereinzelt psychotherapeutische E rfahrungen vor [Ü berblick bei Ehrhardt und Plattner, 1999]. Bisher konnte nachgewiesen werden, daß mit $\mathrm{H}$ ilfe eines verhaltenstherapeutischen A ktivitätenaufbaus die D epressivität bei beginnender A D erheblich verbessert werden kann [Teri, 1994; Teri et al., 1997]. Wie Studien aus dem stationären Bereich zeigen, können die zu einer selbständigen L ebensführung notwendigen Fertigkeiten mit verhaltenstherapeutischen Techniken gefördert werden [G ötestam und M elin, 1990; M CE voy und Patterson, 1986].

Erstmals liegt mit dem von Ehrhardt und seinen Kollegen [E hrhardt et al., 1998; E hrhardt und Plattner, 1999] entwickelten Verhaltenstherapeutischen Kompetenztraining (V KT) eine aus bisherigen Forschungsergebnissen abgeleitete psychotherapeutische A rbeitsgrundlage für ambulante oder stationär aufgenommene Patienten mit einer beginnenden A D vor.

$\mathrm{D}$ as Programm zielt über die Verbesserung depressiver Verstimmungen und eine individuell angepaßten A ktivierung der Patienten auf eine Stabilisierung der kognitiven Symptomatik hin. D as V K T ist für ein E inzel- oder K leingruppensetting mit 12-40 50minütigen Therapieeinheiten konzipiert und setzt verhaltenstherapeutische Standardverfahren ein. Die Therapiebausteine des VKT sind in besonderem M aße für die Behandlung von AD-Patienten geeignet und wurden entsprechend der B edürfnisse der Zielpopulation modifiziert. In den ersten Sitzungen wird eine ausführliche A namnese erhoben sowie eine Verhaltens- und Bedingungsanalyse erstellt. Die weiteren Sitzungen beinhalten obligatorisch die Förderung als angenehm erlebter A ktivitäten und psychoedukative E lemente, je nach Indikation Streßmanagement, das E inüben sozialer Kompetenz oder die Modifikation depressiogener Kognitionen.

\section{Falldarstellung}

\section{A ktuelle Symptomatik zu Therapiebeginn}

Der 53jährige freundlich zugewandte Patient berichtet, seit etwa zwei Jahren «stimme etwas nicht mehr mit ihm». E r habe zunächst unter allgemeinem Energieverlust und großer $M$ üdigkeit gelitten. $D$ anach habe er eine $A$ bnahme seines $G$ edächtnisses, seines Konzentrationsvermögens und seiner Koordination bemerkt. E r müsse sich A bsprachen von Terminen sofort notieren und könne in Gesprächen bei raschem Themenwechsel häufig nicht mehr folgen. Die O rientierung in fremder U mgebung falle ihm im G egensatz zu früher erheblich schwerer. A uch in seinem B eruf habe er Probleme, die er bisher mit H ilfe kompetenter $\mathrm{M}$ itarbeiter habe kompensieren können. Er habe A ngst vor der weiteren Entwicklung und den Folgen seiner $G$ edächtnisprobleme und sei deshalb an einer psychotherapeutischen Behandlung sehr interessiert. $\mathrm{N}$ ach einer stationären A bklärung der dementiellen Symptomatik wurde dem Patienten von dem behandelnden Klinikarzt zu einer ambulanten Verhaltenstherapie geraten.

Der Patient bemühte sich im E rstkontakt darum, heiter und gelassen zu erscheinen. Im weiteren Verlauf der Therapie machte er einen belasteten Eindruck, war zum Teil ratlos, im A ffekt erschien er aber auslenkbar und schwingungsfähig. Er war wach, bewußtseinsklar, jedoch nur mäßig örtlich, aber zeitlich und zur Person gut orientiert. Im formalen Denken zeigte der Patient die Tendenz zu perseverieren und redete häufig am Thema vorbei. Inhaltlich stand die Sorge um die Gedächtnisstörung im Vordergrund. Psychotische Ich- oder Wahrnehmungsstörungen fanden sich nicht. Deutlich waren die kognitiven und mnestischen B eeinträchtigungen. I $m$ Vordergrund stand eine Störung der Aufmerksamkeit und der Konzentration, des Kurzzeitgedächtnisses, der Wortfindung und -flüssigkeit sowie visokonstruktiver und praktischer Leistungen. H inweise auf Suizidalität ergaben sich nicht.

Somatischer B efund: D er sportliche und durchtrainierte Patient befand sich in einem sehr guten A llgemeinzustand ( $\mathrm{Ge}$ wicht: $84 \mathrm{~kg}$ bei 1,80 m K örpergröße, Blutdruck: 140/80, Puls: 58). Die allgemeine somatische und klinisch-neurologische U ntersuchung ergab keine pathologischen B efunde.

Zusatzuntersuchungen: I m Neuroimaging fanden sich erweiterte innere und äußere Liquorräume. E ine Positronen-E mis- 
sions-Tomographie ergab einen ausgeprägten $\mathrm{H}$ ypometabolismus beidseits temporoparietal und geringer auch prämotorisch mit relativer Aussparung der Zentralregionen, der typisch für das Vorliegen einer AD war. Der Befund der $L$ iquoruntersuchung war unauffällig, insbesondere bestanden keine $\mathrm{H}$ inweise auf eine entzündliche Erkrankung. D ie übrigen laborchemischen U ntersuchungen erbrachten keine Befunde im Sinne einer metabolischen, endokrinologischen oder anderen internistischen G runderkrankung, die eine sekundäre $D$ emenz nahelegen würden.

Psychometrie und D iagnose: Es zeigte sich bei einem geschätzten Verbal-IQ von 107 eine dementielle Symptomatik. M ini-M ental-State: 23 von 30 Punkten, Sisco/Sidam-Score: 26 von 55 Punkten (Verdacht auf Demenz), SK T (SyndromK urztest) 22 von 27 Punkten (mittelschweres organisches Psychosyndrom).

A ufgrund des typischen klinischen Verlaufes, des M usters kognitiver D efizite in der neuropsychologischen Testung sowie des charakteristischen PET-B efundes bei fehlenden $\mathrm{H}$ inweisen auf eine vaskuläre, entzündliche oder durch eine andere Primärerkrankung bedingte $G$ enese wurde nach den NINCDS-A D R DA K riterien [M CK hann et al., 1984] die D iagnose einer wahrscheinlichen A D gestellt. Es wurde ein leichtes bis mittelgradiges dementielles Syndrom bei Verdacht auf A Izheimer-D emenz (ICD-10 F00,0, DSM-IV 290,1) diagnostiziert.

\section{B iographische Vorgeschichte}

Der Vater $(* 1925)$ des Patienten sei, als der Patient ein Jahr alt war, im Krieg gefallen. Sein Stiefvater (*1932), mit dem die M utter (*1926) ab dem sechsten L ebensjahr des Patienten zusammenlebte, war ein Polier, der als Montagearbeiter oft monatelang abwesend war. Der Stiefvater habe sich nicht in die Situation seines Stiefkindes einfühlen können, es habe keinen emotionalen Austausch gegeben. Die Mutter sei ängstlich und scheu gewesen. E motionale W ärme und körperliche Nähe erhielt der Patient durch die M utter vor allem bei guten L eistungen in der Schule. E ine wichtige B ezugsperson sei die $\mathrm{G}$ roßmutter mütterlicherseits gewesen, bei welcher der Patient zusammen mit der Mutter bis zum 6. Lebensjahr in Frankreich lebte. Er wurde von der Großmutter bevorzugt behandelt und häufig beschenkt. Der 5 Jahre jüngere Stiefbruder, ein Schlossermeister, wird vom Patienten als zögerlich und ängstlich beschrieben. D ie B eziehung zu diesem sei eher distanziert. Im Vorschulalter habe der Patient unter dem ausgrenzenden Verhalten und den $\mathrm{H}$ änseleien der französischen $\mathrm{K}$ inder gelitten. $\mathrm{E} r$ reagierte darauf häufig sehr wütend und habe zusammen mit seinem Cousin zurückgeschlagen. Später sei er in der Schule am liebsten in der R olle des «U nauffälligen» gewesen. Er habe sehr unter den im Vergleich zu den Schulkameraden ärmlichen Verhältnissen Zuhause gelitten, die ihn z.B. gezwungen hätten, Frauenkleider zu tragen. E r habe keine speziellen Freunde gehabt, sei aber in den $\mathrm{K}$ las- senverband integriert gewesen. Von den L ehrern sei er wegen guter N oten häufig bevorzugt behandelt worden. Der Patient ging gerne zur Schule und zeigte mit zunehmender $D$ auer des Schulbesuchs gute L eistungen. Es sei dem Patienten schon immer schwergefallen, über $\mathrm{G}$ efühle zu sprechen. In der Kernfamilie sei Sexualität ein Tabuthema gewesen. D ie einzige ernstzunehmende sexuelle B eziehung habe zu seiner $E$ hefrau bestanden, die er 1972 heiratete. Seit ungefähr fünf Jahren lehne sie den $\mathrm{G}$ eschlechtsverkehr jedoch ab, was er aber akzeptiere, um die Ehe nicht aufs Spiel zu setzen. A ufgrund seiner $\mathrm{K}$ rankheit könne der Patient seinen prämorbid von inm als sehr stützend erlebten B eruf als kaufmännischer A ngestellter nicht mehr ausüben und stehe vor der Frühberentung. In der Vergangenheit habe es immer wieder Schwierigkeiten mit der Ehefrau - einer 5 Jahre jüngeren Verlegerin gegeben, die schon vor Beginn der E rkrankung dazu geneigt habe, den Patienten zu kontrollieren und zu bevormunden. Ü berhaupt habe er manchmal den $\mathrm{E}$ indruck gehabt, die Frau habe inn nur wegen seiner guten beruflichen Leistungen geschätzt. D ie dominante, ehrgeizige, konservative und engagierte $E$ hefrau sei aber gerade jetzt eine wichtige Stütze. Vor allem aber an seinen Söhnen (17 und 22 Jahre alt) hänge er sehr und habe mit innen an den Wochenenden stets zahlreiche sportliche A ktivitäten unternommen. $\mathrm{D}$ ie beiden $\mathrm{K}$ inder leben noch im gemeinsamen $\mathrm{H}$ aushalt, seien aber gerade dabei, sich vom E Iternhaus abzulösen. Die ersten G edächtnisprobleme seien etwa vor 2 Jahren aufgetreten.

Verhaltens- und B edingungsanalyse

Verhaltenstherapeutische $\mathrm{K}$ rankheitskonzepte gehen davon aus, daß E rkrankungen R eaktionen auf vier E benen des O $\mathrm{r}$ ganismus zeigen können: Neben der psycho-physiologischen $E$ bene sind auch Verhalten, E motionen und Kognitionen in den Krankheitsprozeß involviert. A lle Ebenen und ihre Wechselwirkungen spielen sowohl bei der Entstehung als auch bei der Aufrechterhaltung der Erkrankung und ihrer Symptomatik eine R olle.

\section{D arstellung auf M ikroebene}

\section{Situation}

B eim $A$ bendessen mit Freunden kann der Patient dem lebhaften $\mathrm{G}$ espräch nicht immer folgen. Er möchte antworten, hat aber schon vergessen, was der andere gesagt hatte.

\section{Verhalten}

- E motional: D er Patient fühlt sich deprimiert, gequält, unfähig und hilflos.

- Kognitiv: «D ie anderen sprechen so unzusammenhängende Sätze, daß kein M ensch mitkommen kann».

- Körperlich: Der Patient berichtet über Nervosität und Schwitzen. 
- H andelnd: Der Patient zieht sich zurück und wird ungewohnt still und passiv.

\section{Konsequenzen}

- Kurzfristige Konsequenz: D ie unangenehme Situation verliert an Aversivität (Vermeidung, B eendigung des aversiven Stimulus).

- L angfristige Konsequenz: Patient nutzt seine kognitiven R essourcen nicht, die durch $\mathrm{N}$ icht-G ebrauch schneller verloren gehen.

\section{D arstellung der M akroebene}

A us seinen frühkindlichen Erfahrungen heraus entwickelte der Patient das B edürfnis nach $G$ eborgenheit, A nerkennung und W ärme. D iese B edürfnisse wurden früher nur durch sehr leistungsorientiertes Verhalten des Patienten verstärkt. Die Erkrankung an dem dementiellen Syndrom steht in besonders krassem Gegensatz zu der kindlichen Überlebensregel des Patienten. D iese lautet: «N ur wenn ich überdurchschnittliche Leistungen bringe, erhalte ich ausreichend A nerkennung und vermeide meine A ngst vor $A$ blehnung und $L$ iebesverlust.» Der Patient hat daher keinerlei geeignete CopingStrategien für den U mgang mit dieser E rkrankung zur Verfügung. A lle prämorbiden Selbstwertquellen, wie berufliche A rbeit, A utofahren und Tennisspielen, kann der Patient nicht mehr nutzen. Wegen der $K$ rankheit mußte der Patient seine A rbeit aufgeben. Seither ist der Patient in einen Zustand zunehmender Bemutterung und Fremdbestimmung durch seine Frau geraten. Durch die dementielle Erkrankung kommt es zu weiterer $\mathrm{H}$ andlungsunfähigkeit und Passivität, zum Zusammenbruch der Selbstwertregulation und zum sozialen $\mathrm{R}$ ückzug. Vorhandene R essourcen bleiben ungenutzt.

Z usammenfassend läßt sich folgendes SO RK -Schema erstellen Symptomauslösende Lebenssituation (S) - Erkrankung an einem dementiellen Syndrom.

Organismusvariable (O) - objektivierbare B eeinträchtigung des Gedächtnisses; Entwicklung geeigneter Coping-Strategien gelang aufgrund der L erngeschichte nicht.

Reaktion ( $R$ ) - Passives Sich-Fallenlassen in die E rkrankung und sozialer $R$ ückzug.

Konsequenzen (K) - Kognitiver Status und alltagsrelevante Fertigkeiten sind stärker beeinträchtigt, als es nach dem Stadium der E rkrankung zu erwarten wäre.

Therapieziel und Prognose

1. Stabilisierung der kognitiven Symptomatik.

2. B ewältigung der B elastungen, die sich aus der E rkrankung selbst sowie aus der Konfrontation mit der D iagnose ergeben. A ufbau von Selbstakzeptanz für die eigenen Leistungseinbußen und Veränderung der L ebensgestaltung im Zusammenhang mit der G edächtnisproblematik.

3. M obilisierung vorhandener persönlicher R essourcen; A ufbau mehrerer erfüllender A ktivitäten, die trotz der Gedächtnisproblematik ausgeübt werden können.
D urch die Bearbeitung von Ziel 2 und Ziel 3, sollte Ziel 1 indirekt erreicht werden. Ziel 3 wurde durch direktes A rbeiten an der Modifikation dieser Verhaltensweisen behandelt. U nter Berücksichtigung der starken Leistungsorientierung des Patienten erscheint die B earbeitung von Punkt 2 ebenfalls besonders wichtig.

A Is prognostisch ungünstig war der zu erwartende progrediente Verlauf der dementiellen Symptomatik zu betrachten. Prognostisch günstig zu bewerten war die Unterstützung durch die E hefrau und die große M otivation und der L eidensdruck des Patienten. Ä ußerer B eweggrund für die Therapie waren die auftretenden beruflichen Schwierigkeiten. Innerer B eweggrund waren die massiven Ä ngste und Sorgen, die sich mit B eginn der E rkrankung eingestellt hatten.

\section{Behandlungsplan}

Der Patient nahm an einer ambulanten verhaltenstherapeutischen $\mathrm{E}$ inzelbehandlung teil, die innerhalb von 8 Wochen mit 15 Therapiestunden durchgeführt wurde. Die M ethode richtete sich nach dem Therapiekonzept des Verhaltenstherapeutischen Kompetenztrainings für Patienten mit einer beginnenden A D von E hrhardt und Kollegen [1998].

$\mathrm{D}$ as $\mathrm{VKT}$ ist für ein $\mathrm{E}$ inzel- oder $\mathrm{K}$ leingruppensetting mit 12-40 50minütigen Therapieeinheiten konzipiert und setzt sich aus 6 Therapiemodulen zusammen: (1) Therapieplanung und Verhaltensanalyse, (2) Psychoedukation, (3) A ktivitätenaufbau, (4) Streßmanagement, (5) Förderung sozialer Kompetenz, und (6) Modifikation dysfunktionaler Kognitionen. D ie E lemente (1) - (3) sind obligatorisch, während (4) - (6) je nach Schwerpunkt und Dauer der Therapie variabel eingesetzt werden. Für den Patienten wurden die ersten drei A rbeitstechniken und Punkt (6) ausgewählt.

Der Schwerpunkt lag zunächst im Aufbau der Selbstakzeptanz im Z usammenhang mit einer dementiellen Erkrankung sowie mit dem großen Leistungsanspruch des Patienten, und im A ufbau alternativer A ktivitäten. Z um A ufbau von Selbstakzeptanz wurden kognitive Techniken eingesetzt. D er A ktivitätenaufbau sollte mittels Selbstkontrollstrategien mit gezielter Selbstbeobachtung und Selbstbewertung und positiver Verstärkung von Teilerfolgen durchgeführt werden. Im R ahmen der Psychoedukation sollten zwei B ehandlungseinheiten zusammen mit der Ehefrau des Patienten durchgeführt werden.

\section{Therapieverlauf}

Der Patient machte im Erstkontakt einen unnahbaren und eher verschlossenen E indruck, gab sich aber heiter und gelassen. D em Therapeuten fiel auf, daß er M ühe hatte, die bestehende Problematik des Patienten nicht ebenfalls zu verdrängen und sich auf die offensichtlich dahinterliegenden Proble- 
Tab. 1. Themen der Therapiesitzungen und angewandte $M$ ethoden

\begin{tabular}{lll}
\hline Stunde & Thema der Sitzung & A ngewandte M ethode \\
\hline 1 & Psychoedukation/E rstgespräch & Nondirektive E xploration \\
2 & Psychoedukation/A namneseerhebung & Verhaltensdiagnostik-System [SU LZ, 1992] \\
3 & Psychoedukation/Nerhaltensanalyse & SO R K -M odell \\
4 & Psychoedukation & Interaktive didaktische M ethoden \\
5 & L oslassen/A bschiednehmen/Trauern & Fokussierung der E motionen \\
6 & L oslassen/A bschiednehmen/Trauern & Fokussierung der E motionen \\
7 & U mgang mit der K rankheit & Kognitive U mstrukturierung \\
8 & U mgang mit der K rankheit & R ollenspiel \\
9 & Paargespräch & Fördern des D ialoges \\
10 & Paargespräch & Fördern des D ialoges \\
11 & R egistrierung des A ktivitätsniveaus & Liste angenehmer A ktivitäten - A D \\
12 & A ktivitätenaufbau & Tagesprotokolle / Selbstverstärkung \\
13 & A ktivitätenaufbau & Tagesprotokolle / Selbstverstärkung \\
14 & A ktivitätenaufbau & Tagesprotokolle / Selbstverstärkung \\
15 & A bschluß der Therapie & Interaktiver D ialog \\
\hline
\end{tabular}

me einzulassen. Im weiteren Verlauf der Therapie wirkte der Patient zunächst bedrückt und ratlos, jedoch gut motiviert. Das hervorstechendste Merkmal seiner Persönlichkeit war seine starke Leistungsorientierung, der Wunsch, möglichst überall perfekt zu sein. In diesem Zusammenhang gelang es dem Patienten nur zögernd, die Expertenrolle des Therapeuten zu akzeptieren.

I $m$ therapeutischen Vorgehen wurde ein der A D angepaßter Stil gewählt. Informationsvermittlung und Therapiegestaltung wurden besonders stark strukturiert. Die Grundgedanken der Therapie wurden immer wieder transparent gemacht. Der Therapeut bemühte sich um kurze und prägnante A usführungen und benutzte bildhafte B eispiele zur Veranschaulichung. D ie Therapiematerialien sind in besonderem $\mathrm{M}$ aße für A D-Patienten aufbereitet. Die verwendeten A rbeitsvorlagen sind besonders übersichtlich gestaltet und in G roßschrift gedruckt.

D ie Strukturierung der Therapie verdeutlicht Tabelle 1. D er erste Termin stand im Zeichen gegenseitiger Information: von Seiten des Therapeuten bezüglich der angebotenen Therapiestrategie, von Seiten des Patienten zu seinen Erwartungen und B eschwerden. E s erfolgte eine A ufklärung des Patienten und seiner Frau darüber, daß die psychotherapeutische $M$ aßnahme der möglichst weitgehenden A usschöpfung der noch vorhandenen R essourcen des Patienten dienen soll.

In der nächsten Stunde wurde gemeinsam die A namnese erhoben, welche die Ehefrau fremdanamnestisch bestätigen konnte. In der dritten Stunde stand das E rstellen einer Verhaltensanalyse im Mittelpunkt. Dabei wurde immer wieder deutlich, daß der Patient die durch die K rankheit entstandenen $E$ inschränkungen nicht wahrhaben wollte. Seine R eaktion auf diese Verluste war zunächst der A ufbau einer Fassade: er wich A ntworten aus und stritt seine Vergeßlichkeit ab. Daher war es wichtig, dieses Vermeidungsverhalten zu modifizieren. B esonderen $\mathrm{R}$ aum in den ersten $\mathrm{G}$ esprächen nahmen immer wieder R eflexionen über die A Izheimersche E rkrankung ein. D er Patient war schon vor B eginn der Therapie von ärztlicher Seite über seine D iagnose informiert worden.

In den folgenden Stunden gelang es der Psychoedukation, dem Patienten zu verdeutlichen, daß die A D im Frühstadium keineswegs ausschließt, viele B ereiche der persönlichen Lebensführung weiterhin selbständig zu gestalten. G erade unter dem A spekt seiner starken L eistungsorientierung litt der Patient besonders unter seiner Frühpensionierung und dem Verlust der Fähigkeit zum A utofahren und Tennisspielen.

D em Patienten wurde durch Fokussierung der E motionen die Gelegenheit gegeben, den Abschied von den bisherigen Selbstwertquellen zu betrauern. Dem Patienten war es nicht möglich, durch Weinen dem D ruck seiner E motionen nachzugeben. Er wirkte jedoch am Ende der Stunden emotional sichtlich erleichtert und war nachfolgend in der Lage, alternative A ktivitäten aufzubauen.

A Is Vorbereitung für eine veränderte L ebensführung war es von Bedeutung, die Selbstakzeptanz des Patienten in bezug auf die E rkrankung wiederherzustellen. E in erster Schritt dazu war zunächst die Erkenntnis, wie sehr das heutige Selbstbild noch von kindlichen $\mathrm{G}$ rundannahmen geprägt wird. E inige seiner Lebenskonzepte wurden herausgearbeitet. In der kognitiven Restrukturierung wurden die dysfunktionalen Schemata bezüglich des leistungsorentierten Weltbildes ( «Wenn ich bei meiner A rbeit versage, dann bin ich als ganzer M ensch ein Versager») modifiziert. Die zugrundeliegende kindliche Ü berlebensregel wurde so lange kritisch hinterfragt, bis der Patient sie umformulieren konnte. Die kindliche Grundannahme lautete: «Nur wenn ich perfekt den N ormen eines $M$ annes entspreche, $d$.h. überdurchschnittlich beruflich aktiv und emotional zurückhaltend bin, kann ich durch die U mwelt anerkannt werden.» A Iternative Gedanken wie «auch ohne L eistung kann ich A nerkennung bekommen» wurden mittels sokratischem Dialog gefunden und führten zu G efühlen von E rleichterung und Stimmungsverbesserung. 
Tab. 2. Testergebnisse im A DA S-COG

\begin{tabular}{llllll}
\hline Test & $\begin{array}{l}\text { Maximaler } \\
\text { Wert }\end{array}$ & $\begin{array}{l}\text { T1 } \\
\text { (Woche 1) }\end{array}$ & $\begin{array}{l}\text { T2 } \\
\text { (Woche 5) }\end{array}$ & $\begin{array}{l}\text { T3 } \\
\text { (Woche 9) }\end{array}$ & $\begin{array}{l}\text { T4 } \\
\text { (Woche 19) }\end{array}$ \\
\hline $\begin{array}{llllll}\text { 1. Freie R eproduktion } \\
\text { 7. Wort-Wiedererkennung }\end{array}$ & 10 & 7 & 5 & 4 & 4 \\
Summe: G edächtnis & 12 & 5 & 4 & 6 & 5 \\
& 22 & 12 & 9 & 10 & 9 \\
2. O rientierung & 8 & 4 & 0 & 1 & 0 \\
3. Vorstellungsvermögen & 5 & 1 & 1 & 1 & 1 \\
4. A bzeichnen & 5 & 3 & 3 & 3 & 3 \\
5. A nweisungen & 5 & 3 & 3 & 2 & 2 \\
$\begin{array}{l}\text { 6. B enennen } \\
\text { 8. E rinnern der Prüfungs- }\end{array}$ & 5 & 2 & 4 & 5 & 4 \\
$\quad$ anweisungen & 5 & 0 & 4 & 1 & 2 \\
19. Sprachausdruck & 5 & 0 & 2 & 1 & 1 \\
20. Sprachverständnis & 5 & 0 & 2 & 2 & 2 \\
21. Wortfindungsstörungen & 5 & 1 & 3 & 3 & 2 \\
Summe: Kognitive Items außer & & & & & \\
$\quad$ G edächtnis & 48 & 14 & 22 & 19 & 17 \\
G esamt: Kognitiver B ereich & 70 & 26 & 31 & 29 & 26 \\
\hline
\end{tabular}

E in zweiter Schritt zur Verbesserung der Selbstakzeptanz war das E ingestehen seiner E rkrankung, auch seiner U mwelt gegenüber. $\mathrm{M}$ it dem Patienten wurden verschiedene $\mathrm{A}$ rten des Verhaltens gegenüber anderen diskutiert. D er Patient gelangte schließlich zu der Ü berzeugung, daß es ihn entlasten könnte, offen über seine $K$ rankheit sprechen zu können. $M$ it dem Patienten wurden in Rollenspielen konkrete Dialoge eingeübt. D em Patienten gelang daraufhin die A nwendung im A IItag. So war es inm möglich, mit einem Freund auch über die $K$ rankheit zu sprechen. $M$ it der außerordentlich verständnisvollen und unterstützenden R eaktion des Freundes hatte der Patient nicht gerechnet. Neben der emotionalen Erleichterung hatte dies auch den Effekt, dem sozialen Rückzug des Patienten entgegenzuwirken.

$D$ a der Patient nach der Frühpensionierung nun den $G$ roßteil der Z eit zu Hause verbrachte, wurde eine Integration der E hefrau in die Therapie angestrebt. Es ging darum, den Dialog zwischen den Partnern zu fördern. Im G espräch entstand der E indruck, daß es der E hefrau darum ging, die Kontrolle über das, was mit ihrem M ann vor sich ging, zu behalten. Sie ergriff häufig für ihren $M$ ann das Wort und unterbrach inn. Sie entwickelte zunehmend Verständnis für ungünstige Interaktionsmuster innerhalb der Partnerschaft - nämlich zunehmende Ü bernahme von den Patienten betreffenden E ntscheidungen -, nachdem sie von dem Therapeuten auf diese dysfunktionalen Verhaltensweisen vorsichtig hingewiesen worden war.

A uf der G rundlage einer speziell für A D -Patienten geeigneten Liste angenehmer A ktivitäten wurden verschiedene A ktivitäten geplant. Der Patient wurde vom Therapeuten immer wieder auf seine Kompetenzen hingewiesen. $M$ it dem Freund ging er nun regelmäßig zum Schwimmen. E r unternahm täg- lich Spaziergänge und ordnete seine Fotos in A Iben ein. D as Selbstbewußtsein und die Selbstakzeptanz erschienen durch diese neuen $\mathrm{M}$ öglichkeiten selbstverantwortlichen $\mathrm{H}$ andelns gestärkt.

Gegenstand der letzten Stunde waren wahrscheinliche zukünftige $\mathrm{K}$ risen und Belastungen und die $\mathrm{M}$ öglichkeit des U mgangs. Darüber hinaus wurde auf $\mathrm{H}$ ilfsmöglichkeiten durch A Izheimer-G esellschaften aufmerksam gemacht.

Kognitive Parameter im Verlauf der Therapie

D ie Veränderungsmessung des Schweregrades kognitiver D emenzsymptome erfolgte mittels des Kognitiven L eistungstests der A lzheimer's Disease A ssessment Scale (A DA S-COG) [Ihl und Weyer, 1993]. Die Testung erfolgte nicht durch den Therapeuten.

Der A DA S-COG enthält 11 U ntertests, welche B eeinträchtigungen in Gedächtnisleistungen und in praktischen, handlungsorientierten Leistungen sowie Beeinträchtigungen der $O$ rientierung und Sprache erfassen. D ie U ntertests 1- 8 bilden den aktiven Testteil, die U ntertests 19-21 dienen der Verhaltensbeobachtung - nämlich der sprachlichen Beeinträchtigung - während der Untersuchung. Ein erhöhter Wert im A DA S-COG bedeutet eine Verschlechterung der kognitiven Parameter.

Die Meßzeitpunkte lagen zu Therapiebeginn (T1), in der $M$ itte (T 2 nach 4 Wochen), am E nde (T 3 nach 8 Wochen) und 10 Wochen nach B eendigung der Therapie (T4).

In Tabelle 2 sind die Testergebnisse des Patienten des A DA SCOG zu den 4 M eßzeitpunkten wiedergegeben. D er G esamtwert zeigt einen stabil gebliebenen Wert zwischen erstem M eßzeitpunkt T1 und K atamnese. Zum M eßzeitpunkt T2 er- 
Abb. 1. Verlauf der A DA S-COG G esamtwerte mit initialer Verschlechterung und $\mathrm{R}$ ückkehr zum A usgangspunkt.

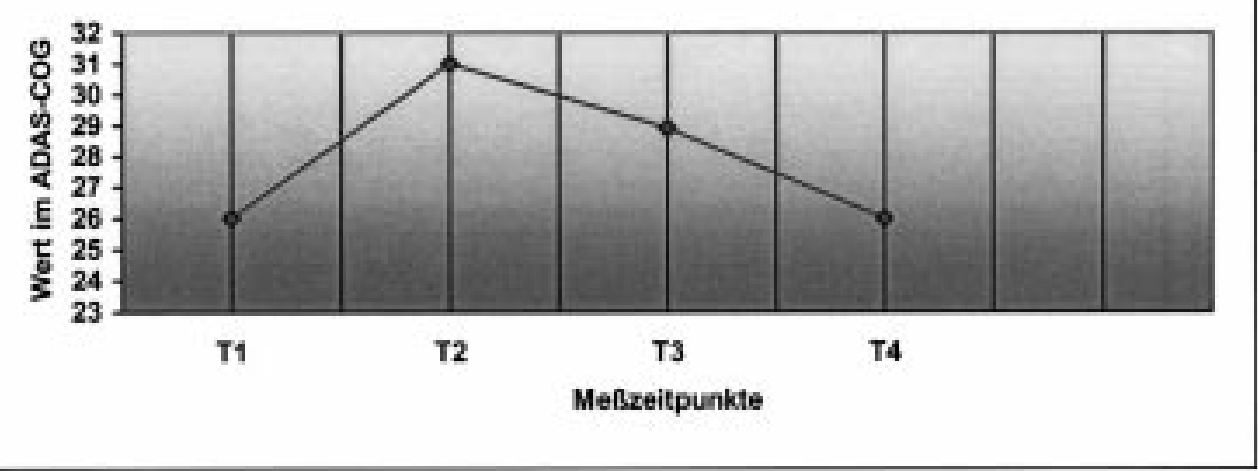

gab sich ein erhöhter Wert, der über den noch leicht erhöhten Wert bei M eßzeitpunkt T 3 wieder auf den A usgangswert abfiel. Der Verlauf des A DA S-COG -G esamtwertes wird durch A bbildung 1 verdeutlicht.

In den Tests, die den sprachlichen Bereich abfragen, hat sich der Patient vom Meßzeitpunkt T1 zum Meßzeitpunkt T2 leicht verschlechtert, dieser Wert wurde anschließend weitgehend stabil gehalten. In den U ntertests zu Gedächtnis und O rientierung verbesserte sich der Patient vom M eßzeitpunkt T1 zu Meßzeitpunkt T2 und blieb dann bis zur Katamnese stabil.

\section{Diskussion}

In A nbetracht der bisher noch eingeschränkten Wirksamkeit der medikamentösen Behandlungsstrategien bei einer $A D$ besteht ein großer B edarf an der Entwicklung und dem kontrollierten $\mathrm{E}$ insatz neuer therapeutischer Interventionen.

$\mathrm{N}$ icht nur die Grenzen der herkömmlichen Therapie, sondern auch die der E rkrankung immanenten M erkmale, wie niedriges A ktivitätsniveau, dysfunktionales Coping, Partnerschaftskonflikte und depressive Symptome, weisen den Weg zu einem psychotherapeutischen A nsatz.

D ie Konfrontation mit kognitiven D efiziten wird von A D -Patienten als große B elastung empfunden. In diesen Situationen unterbrechen sie dann häufig befriedigende A ktivitäten. D ie U nterbrechung angenehmer Gewohnheiten unter belastenden B edingungen läßt sich nicht nur als Konsequenz, sondern auch als B edingung zusätzlicher B elastungen betrachten. Der hier beschriebene Fall zeigt, daß eine niedrige Verstärkungsrate auch per se eine B elastung darstellen oder intensivieren kann. Ist die Aufmerksamkeit durch dominante Belastungsthemen absorbiert, sind die Patienten weniger in der L age, in anderen A ktivitätsbereichen erfolgreich zu handeln und in ihren A ktivitäten verstärkt zu werden.

W ie bereits angedeutet, ist die Pathogenese der dementiellen Symptomatik zwar auf die degenerativen Veränderungen in der $\mathrm{H}$ irnrinde zurückzuführen, Auftreten und Schwere der kognitiven Symptome können aber von Faktoren wie emotio- naler Z ustand und allgemeiner A ktivierung moduliert werden. E motionale Faktoren sind für Vorgänge der Informationsverarbeitung und damit für die L eistungsfähigkeit des G edächtnisses von B edeutung [M arkowitsch, 1992].

D as A ktivitätsniveau hat im A Iter außerdem experimentell nachgewiesene Effekte auf die kortikale Plastizität [Swaab, 1991], d.h. auf die Fähigkeit der Hirnrinde, vorhandene Synapsen zu stabilisieren und neue Synapsen zu bilden. Während aktives Verhalten die kortikale Plastizität fördert, stützt Inaktivität neurodegenerative Effekte. Nicht nur im A Iter, sondern auch bei degenerativen $\mathrm{H}$ irnerkrankungen lassen sich morphologische B efunde erheben, die auf plastische R eserven des menschlichen Gehirns schließen lassen [Ü bersicht bei Gehrtz, 1989].

B ei der D emenzbehandlung geht es nicht um eine vollständige Rehabilitation. Die derzeitige Befundlage zeigt, daß ein kognitiver A bbau nicht verhindert werden kann. Es ist daher die Z ielsetzung einer Psychotherapie mit D emenz-Patienten im Frühstadium der E rkrankung, Phasen der Symptomstabilität zu maximieren, bzw. die Symptomprogression allgemein zu verzögern.

In der hier durchgeführten Verhaltenstherapie konnte die kognitive Symptomatik insgesamt stabilisiert werden. Der A usgangswert von 26 im A DA S-CO G [Ihl und Weyer, 1993] wurde in der Katamnese nach viereinhalb M onaten wieder erreicht. D ie Symptomprogression wird von K ramer-G insberg und M itarbeitern [1988] mit jährlich durchschnittlich 7 Punkten im A DA S-COG angegeben. In diesem Licht erscheint das vorliegende $E$ rgebnis durchaus als $E$ rfolg.

Die Stabilisierung der kognitiven Symptomatik wurde im vorliegenden Fall erreicht durch die Modifizierung der dysfunktionalen B ewältigungsstrategien und durch den A ufbau erfüllender Aktivitäten ohne zu über- oder unterfordern. Während der verhaltenstherapeutischen B ehandlung konnte zu B eginn sogar eine Verbesserung der kognitiven Parameter G edächtnis und O rientierung beobachtet werden. D ies könnte als positive A uswirkung einer emotionalen E ntlastung interpretiert werden. $E$ in wichtiges Forschungsziel ist es, diesen kurzfristigen Therapieeffekt über einen längeren Z eitraum hinweg zu optimieren. 
A ufgrund der eingeschränkten Validität von E inzelfallanalysen kann hier von einer erstmaligen Pilot-U ntersuchung einer verhaltenstherapeutischen Intervention ausgegangen werden. Die hier dargestellten U ntersuchungsergebnisse erscheinen hinsichtlich ihrer Quantität nicht ausreichend für eine fun- dierte Beurteilung der Ökonomie des VKT. Eine wichtige Forschungsaufgabe besteht gegenwärtig darin, die N ützlichkeit des V KT durch replizierte E inzelfallanalysen unter variierten B edingungen sowie in einem $\mathrm{G}$ ruppenexperiment zu evaluieren.

\section{Literatur}

Baines S, Saxby P, Ehlert K: R eality orientation and reminiscence therapy. A controlled cross-over study of elderly confused people. B IJ Psychiatry 1987;151:222231.

Bauer, J: M öglichkeiten einer psychotherapeutischen B ehandlung bei $\mathrm{A}$ Izheimer-Patienten im Frühstadium der E rkrankung. Nervenarzt 1997;68:421-424.

Bauer J, Bauer H, Teising M: Psychosomatische A spekte der A Izheimer-Demenz; in $\mathrm{H}$ irsch RD ( $\mathrm{H} \mathrm{rsg}$ ): Psychotherapie bei D emenzen. D armstadt, Steinkopff, 1994, pp 47-62.

Broe GA, Henderson A S, Creasey H, M cCusker E, Korten AE, Jorm A F, Longley W, A nthony J C: A case-control study of $A$ Izheimers disease in A ustralia. Neurology 1990;40:1698-1707.

E hrhardt T, H ampel H, H egerl U, M öller, H-J: Das Verhaltenstherapeutische Kompetenztraining VKT $E$ ine spezifische Intervention für Patienten mit einer beginnenden A Izheimer D emenz. Z G erontol Geriatr 1998;31:112-119.

Ehrhardt T, Kötter U, H ampel H, Schaub A, Hegerl U, Möller, $\mathrm{H}-\mathrm{J}$ : Psychologische Therapieansätze bei Demenz. Z G erontopsychol Gerontopsychiatr 1997;2:

85-98.

Ehrhardt T, Plattner A : Verhaltenstherapie bei Morbus A Izheimer. G öttingen, H ogrefe, 1999.

Folstein M F, Folstein SE, M cH ugh PR: 'M ini-M entalState'. A practical method for grading the cognitive state of patients for the clinical state of patients for the clinician. J Psychiat R es 1975;12:189-198.

Friedland R P, Smyth K, E steban-Santillan C, Koss E, Cole R, Lerner A J, Strauss MS, Whitehouse PJ, Petot $G, R$ owland DY, D ebanne S: Premorbid environmental complexity is reduced in patients with A lzheimers disease (AD) as compared to age and sex matched controls: R esults of a Case-Control Study. Neurobiol A ging 1996;17:122.
Gehrtz $\mathrm{H}-\mathrm{J}$ : Neuronale Plastizität bei degenerativen H irnerkrankungen; in B altes M M, Kohli M, Sames K ( $\mathrm{H}$ rsg): E rfolgreiches $A$ Itern. B edingungen und Variationen. B ern, H uber, 1989, pp 250-253.

Götestam KG, M elin L: The effect of prompting and reinforcement of activity in elderly demented inpatients. Scand J Psychol 1990;31:2-8

H eiss W-D, K essler J, M ielke R, Szelies B, H erholz K : Long-term effects of phosphatidylserine, pyritinol and cognitive training in A lzheimers disease. Dementia 1994:5:88-98.

Ihl R, Weyer G: Alzheimer's Disease A ssessment Scale. Weinheim, B eltz Test, 1993.

K ramer-Ginsberg $E$, M ohs $R C$, A ryan $M$, L obel $D$, Silverman J, D avidson M, D avis K L : Clinical predictor of course for A Izheimer patients in longitudinal study: A preliminary report. Psychopharmacol Bull 1988;24: 458-462.

Markowitsch HJ: Neuropsychologie des G edächtnisses. G öttingen, H ogrefe, 1992.

$M C E$ voy $C L$, Patterson $R L$ : B ehavioral treatment of deficit skills in dementia patients. Gerontologist 1986; 5:475-478.

M CK hann G, D rachmann D, Folstein M, K atzmann R, Price D, Stadlan E M : Clinical diagnosis of A Izheimer's disease: Report of the NINCDS-ADRDA WorkGroup under the auspices of Department of $\mathrm{H}$ ealth and H uman Services Task Force on A Izheimer's disease. N eurology 1984;34:939-944.

McKittrick LA, Camp JC, Black FW: Prospective memory intervention in A Izheimers disease. J Gerontol 1992;47:337-343.

M otomura N, O hkubo F, A sano A, Tomoda J, A kagi $\mathrm{H}$, Seo T: Premorbid behavioral characters in demented patients. Neurobiol A ging 1996;17:122

Patterson MB, Bolger JP: A ssessment of behavioral symptoms in A Izheimer's disease. A Izheimer D isease A ssoc D isorders, 1994;8:4-20.
Pearlson G D, R oss, CA, L ohr WD, R ovner BW, Chase $G A$, Folstein M F : A ssociation between family history of affective disorder and the depressive syndrome of A Izheimer's disease. A m J Psychiatry 1990;147:452456.

Q uayhagen M P, Q uayhagen M : D ifferential effects of family-based strategies on Alzheimer's disease. Gerontologist 1989:29:150-155.

$\mathrm{R}$ abins $\mathrm{P}, \mathrm{Chair} \mathrm{M} \mathrm{PH}$ : Practice guideline for the treatment of patients with A Izheimer's disease and other dementias of late life. A m J Psychiatry 1997;154:1-39. R abins PV, M erchant A, N estadt G : Criteria for diagnosing reversible dementia caused by depression: Validation by 2-year follow-up. Br J Psychiatry 1984; 144:488-492.

Rubin EH, Kinscherf DA: Psychopathology of very mild dementia of the A Izheimer type. A m J Psychiatry 1989:146:1017-1021.

Saß $H$, Wittchen $H-U, Z$ audig $M$ : D iagnostisches und Statistisches M anual Psychischer Störungen D SM -IV. Ü bersetzt nach der vierten Auflage des Diagnostic and Statistical $M$ anual of $M$ ental $D$ isorders der $A$ merican Psychiatric A ssociation. Göttingen, Hogrefe, 1996.

Scanland SG, Emershaw LR : R eality orientation and validation therapy. Dementia, depression and functional status. J G erontol Nurs 1993;6:7-11.

Swaab DF: B rain aging and A Izheimers disease: 'Wear and tear' versus 'use it or lose it'. Neurobiol A ging 1991;12:317-324

Teri $L$ : B ehavioral treatment of depression in patients with dementia. A lzheimer D isease A ssoc D isorders 1994;8:66-74.

Teri L, Logsdon RG, U omoto J, McCurry S: B ehavioral treatment of depression in dementia patients: $A$ controlled clinical trial. J G erontol 1997;52:159-166. Yesavage JA, Westphal J, Rush L: Senile dementia: Combined pharmacologic and psychologic treatment. J A m G eriatr Soc 1981;4:164-171. 